\title{
An Innovative Superconducting Coil Fabrication Method with YBCO Coated Conductors
}

\author{
Katsutoshi MIZUNO \\ Researcher, \\ Masafumi OGATA \\ Senior Researcher, \\ Cryogenic Systems Laboratory, Maglev Systems Technology Division \\ Ken NAGASHIMA, Dr. Eng. \\ Director, \\ Maglev Systems Technology Division
}

\begin{abstract}
YBCO coated conductors attract attention because of their high critical current in high magnetic field conditions, hence calling for a suitable coil fabrication method. One of the critical problems associated with this process, however, is the negative impact on YBCO coil performance due to epoxy impregnation. Epoxy impregnation is an important technique to increase the mechanical strength and thermal conductivity of superconducting coils. However, the bonding strength of epoxy resin is stronger than the delamination strength of YBCO coated conductors in a certain direction. Therefore, thermal stress could damage epoxy impregnated YBCO coils. As a new impregnation material, we have focused on cyanoacrylate resin.
\end{abstract}

Keywords: YBCO coated conductor, superconducting magnet, epoxy, cyanoacrylate

\section{Introduction}

Superconducting applications such as Maglev train will employ high temperature superconductor or HTS in the future. One type of HTS wire in particular called YBCO coated conductor attracts attention because of its high current capacity in magnetic fields. YBCO coated conductors do not require liquid helium because of their high operation temperature. Liquid helium-less cooling means easy-operation of superconducting magnets and lower cooling costs. In the case of a Maglev trains, downsizing of the power source for the cryocooler also means cutting the weight of the overall magnet system.

Nevertheless, YBCO coated conductors have only recently been released onto the market and research into their applications are so far insufficient. The authors therefore set themselves the goal of applying YBCO coated conductor technology to the onboard magnet system of a Maglev train. A magnetic flux density of $5 \mathrm{~T}$ is required for maglev applications. YBCO coil fabrication techniques were therefore investigated followed by the development of a small demonstration YBCO magnet capable of producing a magnetic flux density of $5 \mathrm{~T}$. This paper reports on a new impregnation technique and the basic design of the demonstration YBCO magnet.

\section{YBCO coated conductor}

The critical temperature of YBCO coated conductors is approximately $90 \mathrm{~K}$ and their critical current under high magnetic field conditions is superior to other HTS wires. The YBCO coated conductor consists of a substrate, buffer layers, superconductor layer and stabilizer (see Fig. 1). The substrate is usually Ni-alloy. Therefore, the coated conductor has high-tensile strength. Because of these characteristics, YBCO coated conductors are suitable for high magnetic field applications such as accelerators, NMR or Maglev train onboard magnets.

Coated conductors however are weak against peel force due to their laminated structure [1]. Serious falls in YBCO coil performance caused by epoxy impregnation have been

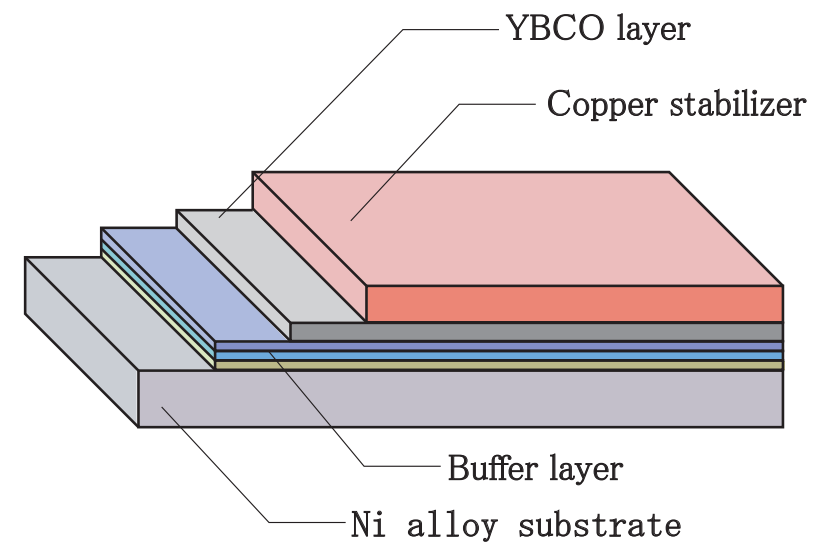

Fig. 1 Typical structure of the YBCO coated conductor

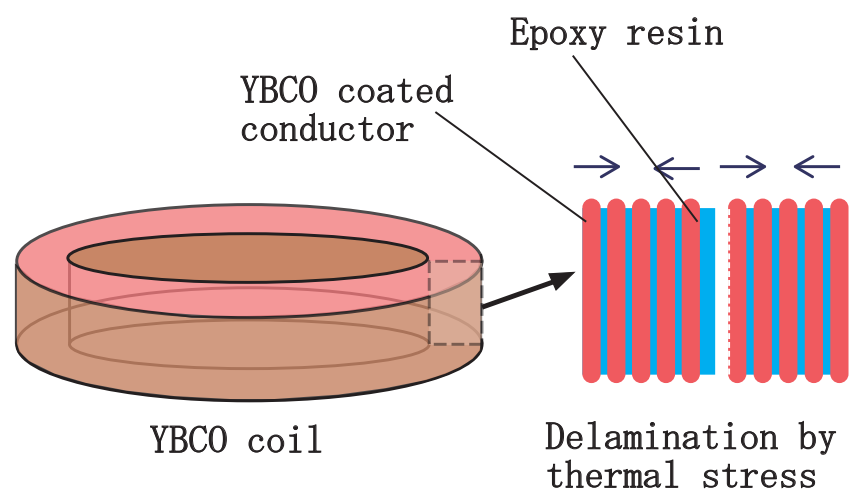

Fig. 2 Image of YBCO coated conductor delamination cased by thermal stress 
reported [2]. The epoxy impregnation technique is common in low temperature superconducting coils. Thermal shrinkage of epoxy resin is greater than in metallic materials such as superconductors. Consequently thermal stress, up to several $\mathrm{MPa}$, is generated by the cooling down process of the epoxy-impregnated coil. In the case of YBCO coils, this thermal stress is strong enough to delaminate the coated conductor and causing serious damage to performance. Coated conductor delamination is illustrated in Fig. 2.

\section{Impregnation of the superconducting coil}

\subsection{Purpose of impregnation}

There are two main reasons for superconducting coil impregnation. One is to increment mechanical strength. Super conducting magnets are exposed to high magnetic fields. Epoxy or other resin impregnation prevents deformation of the coil caused by electromagnetic force.

The other reason is to improve thermal conductivity. HTS magnets are usually cooled by the cryocooler. Insufficient thermal conductivity of the heat path would cause temperature gradation and limit coil performance. In addition, thermal runaway due to over current could cause the HTS wire to burn out. Impregnation material fills the gaps between coil turns and prevents these problems.

\subsection{Selection of impregnation material}

Paraffin and beeswax are also well known impregnation materials in addition to epoxy resin. Paraffin and beeswax have a bonding strength close to zero and do not damage the YBCO coated conductor [2]. Inversely, this also means they cannot maintain the superconducting coil shape and can only be applied as fillers, i.e. these impregnation materials can be employed when the superconducting coil is already placed in the coil case.

The ideal impregnation material for YBCO coils should have moderate bonding strength and low viscosity to be able to fill the small gaps between coil turns. It was decided therefore to concentrate investigations on cyanoacrylate resin as the new impregnation material. Cyanoacrylate resin is famous as an instant adhesive and has fairly low viscosity compared with other adhesives (e.g. $2 \sim 5 \mathrm{mPa} \cdot \mathrm{s}$ for a low viscosity cyanoacrylate resin) whereas the viscosity of epoxy resin is approximately $500 \mathrm{mPa} \cdot \mathrm{s}$. Another characteristic of cyanoacrylate is that the solidification process requires water molecules and cyanoacrylate resin does not cure in vacuum conditions. Hence, vacuum impregnation of cyanoacrylate resin is probable.

\subsection{Bonding strength test of cyanoacrylate resin in liquid nitrogen}

Whereas cyanoacrylate and epoxy resin have similar bonding strengths at room temperature cyanoacrylate resistance to impact or peel force is considered to be weak. The bonding strength of cyanoacrylate at cryogenic temperature is unknown. Bonding strength tests on impregnation materials were carried out in liquid nitrogen. Some test specimens were made to evaluate the peel and tensile

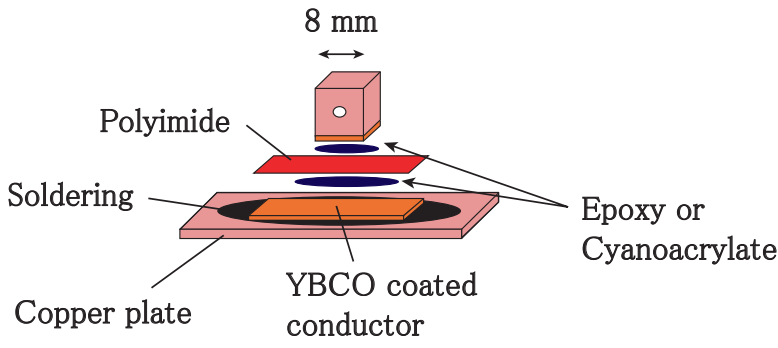

Fig. 3 Schematic drawing of a test specimen for tensile bonding strength tests

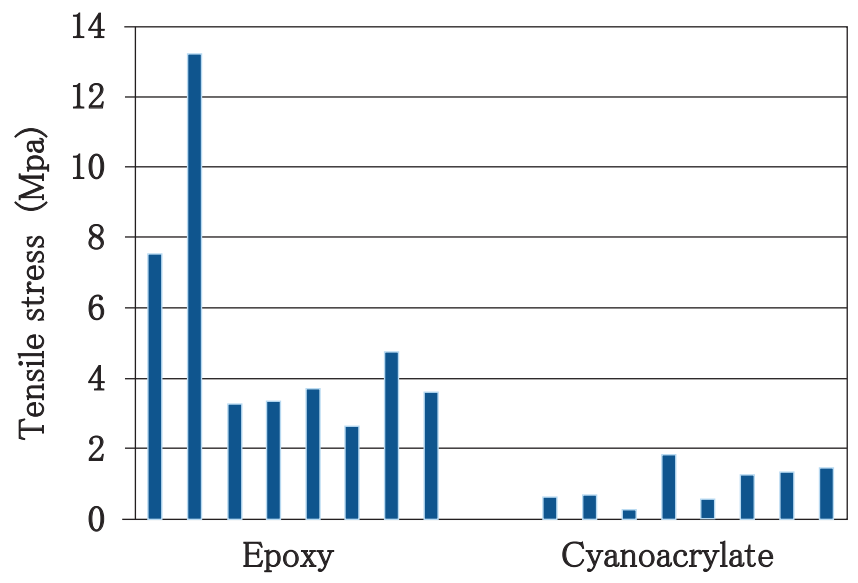

Fig. 4 Tensile bonding strength of epoxy resin and cyanoacrylate resin at $77 \mathrm{~K}$

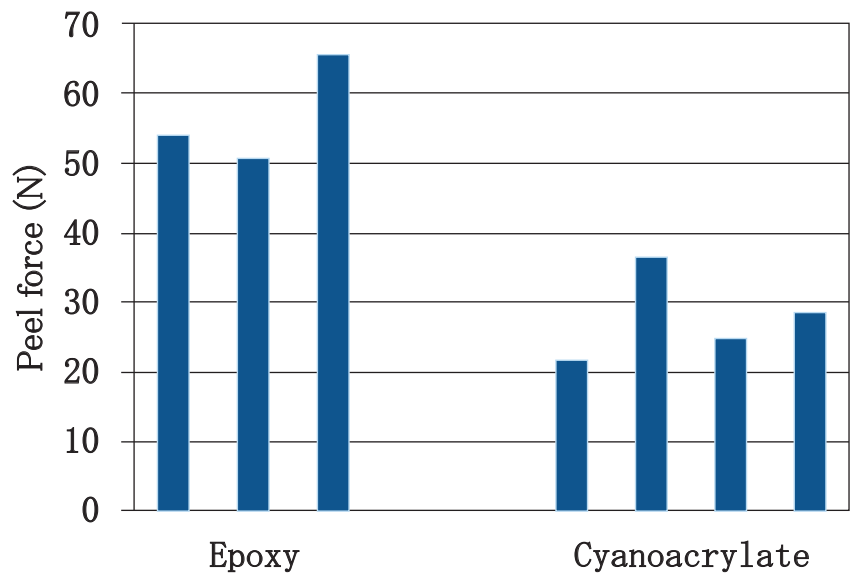

Fig. 5 Peel bonding strength of epoxy resin and cyanoacrylate resin at $77 \mathrm{~K}$

bonding strength of cyanoacrylate and epoxy resin (see Fig. 3). To imitate an actual YBCO coil structure, the adherent surfaces of the test specimens were an actual YBCO coated conductor (Super Power Inc. SCS4050) and polyimide tape.

The bonding areas were $4 \mathrm{~mm} \times 25 \mathrm{~mm}$ on the peel stress test specimen and $4 \mathrm{~mm} \times 8 \mathrm{~mm}$ on the tensile stress test specimen. Several bonding strength tests were performed for each stress mode. The results of the tests are shown in Fig. 4 and Fig. 5. The peel bonding strength of cyanoacrylate resin was less than one-fifth of that of epoxy resin on average (see Fig. 4). The bonding strength of cyanoacrylate resin under peel force was approximately half that of epoxy resin (see Fig. 5). This demonstrates that cya- 
noacrylate resin has moderate bonding strength and is a potential impregnation material for superconducting coils.

\section{Sample YBCO coils for cyanoacrylate impregna- tion}

\subsection{Specifications of sample coils}

Although the bonding stress tests described above indicate that cyanoacrylate resin is weaker than epoxy resin, it is uncertain whether cyanoacrylate resin damages YBCO coils. The mechanism leading to a fall in performance due to epoxy resin impregnation has not yet been clarified. A cyanoacrylate impregnation with actual YBCO coils was therefore carried out.

Two YBCO coils were prepared: sample A and B. Epoxy impregnation was applied to sample A and cyanoacrylate impregnation to sample B coil, for comparison. Figure 6 is a picture of a sample coil and specifications are shown in Table1.

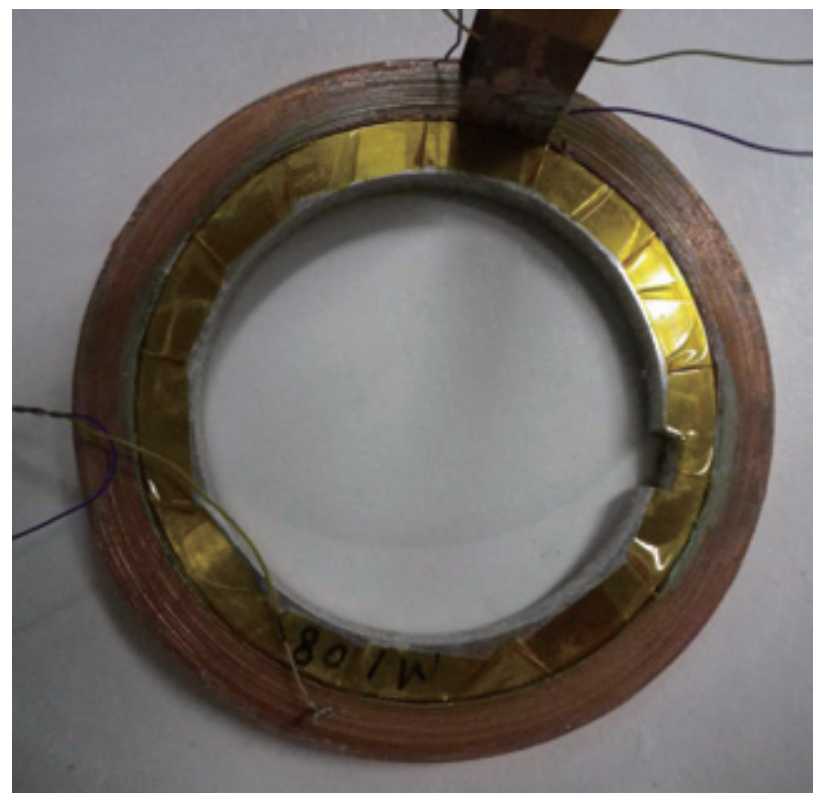

Fig. 6 Picture of the sample YBCO coil for impregnation tests

Table 1 Specifications of the sample coils for impregnation material tests

\begin{tabular}{c|c}
\hline Wire & YBCO(MOCVD)/Hastelloy \\
\hline Width / Thickness & $4 \mathrm{~mm} / 0.1 \mathrm{~mm}$ \\
\hline Ic (77 K, s.f.) & $90 \mathrm{~A}$ \\
\hline Winding & Circular single pancake \\
\hline Outer / Inner diameter & $60 \mathrm{~mm} / 50 \mathrm{~mm}$ \\
\hline Wire length & $7 \mathrm{~m}$ \\
\hline Turns & 40 \\
\hline Bobbin material & Aluminum \\
\hline Insulator & Polyimide tape
\end{tabular}

\subsection{Excitation tests on impregnated YBCO coils}

Each coil was energized until the electric field in the coil exceeded $1 \mu \mathrm{V} / \mathrm{cm}$, i.e. $0.7 \mathrm{mV}$ before and after the impregnation process. Current reaching this voltage is called critical current or Ic. The n-value was also evaluated. The $\mathrm{n}$-value is a parameter indicating the rate at which the electric field rises against current flow. A high n-value indicates rapid rise in electric field, i.e. excellent superconducting properties. An n-value under 10, indicates that the superconductor may be damaged.

Sample A was impregnated with low viscosity epoxy resin (Blenny Giken Ltd, GM6800). Current-Voltage characteristics of sample-A coil are shown in Fig. 7. The critical current of the bare coil was 45.5 A. However, after epoxy impregnation, the critical current was only $27.5 \mathrm{~A}$. The nvalue was also drastically reduced from 27 to 6 and YBCO coil performance degradation was clearly observed.

Sample B coil was impregnated with low viscosity cyanoacrylate resin (Cemedain Co., Ltd., 3000RX). Like epoxy impregnation, sample B was immersed into a cyanoacrylate bath under vacuum conditions for 30 minutes. Then,

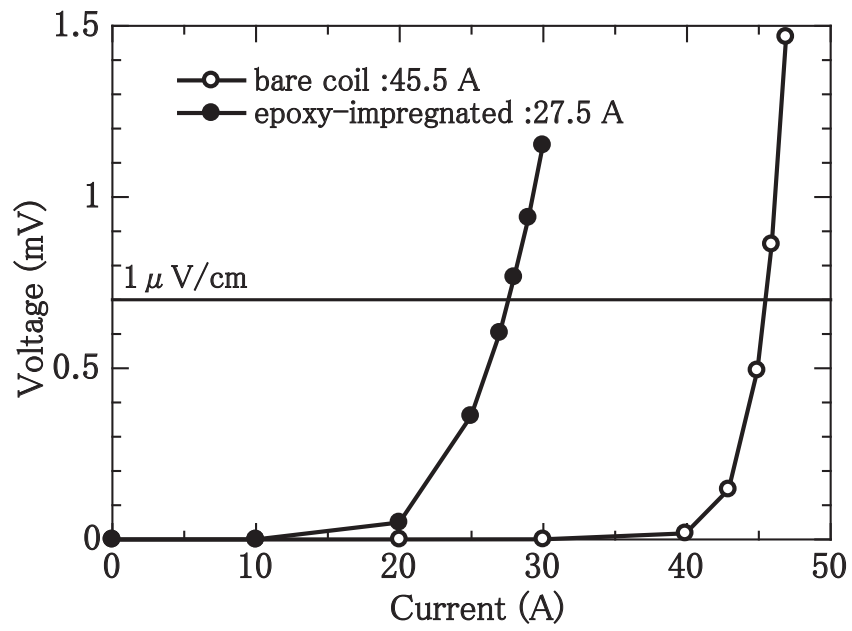

Fig. 7 I-V characteristics of the epoxy-impregnated YBCO coil at $77 \mathrm{~K}$

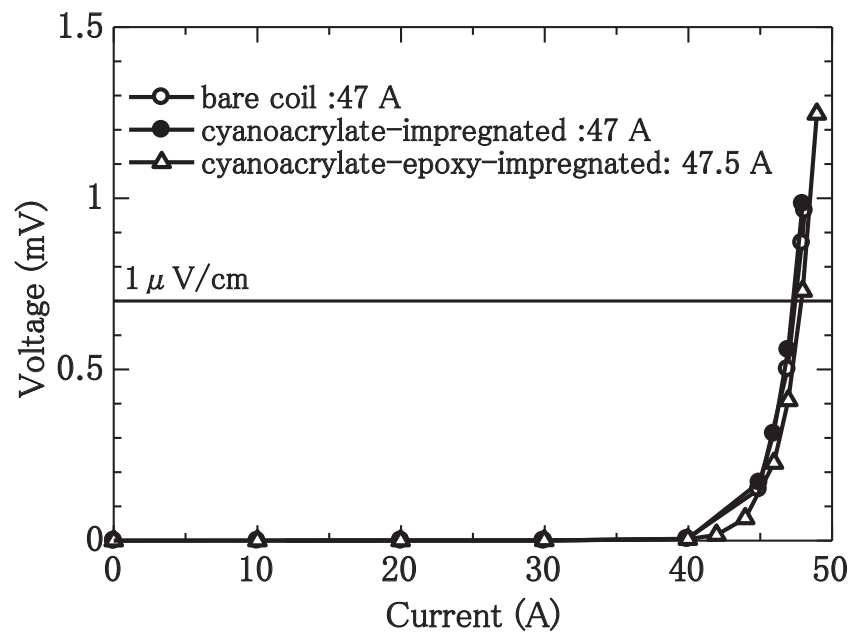

Fig. 8 I-V characteristics of the cyanoacrylate-impregnated YBCO coil at $77 \mathrm{~K}$ 
the coil was exposed to air to cure the cyanoacrylate.

After cyanoacrylate impregnation, the coil was reimpregnated with epoxy resin. The purpose of this process was to confirm cyanoacrylate resin had filled the gaps between coil turns. If this was the case, epoxy resin could not get into the gaps and there would be no loss of performance. Figure 8 shows that Current-Voltage characteristics of sample B coil did not change during the impregnation process.

This cyanoacrylate impregnation technique was tested with different shaped YBCO coils, although no loss of performance was measured.

\section{Five Tesla class YBCO magnet}

A YBCO magnet is developed to demonstrate the advantages of applying YBCO coated conductors to Maglev train on-board superconducting magnets. The demonstration YBCO magnet included the following design principles:

1. Maximum magnetic flux density set to $5 \mathrm{~T}$, same as actual on-board magnets.

2. The operation temperature is approximately $50 \mathrm{~K}$ to simplify the cooling system and thermal insulation.

The operation temperature and the total mass of the magnet are related. Lower operational temperatures require a larger cryocooler. High operational temperatures also signify a vast number of superconductors because of the limited critical current. Investigations into this relationship revealed an operational temperature of $40 \mathrm{~K}$ to 50 $\mathrm{K}$ made it possible to minimize the total mass of the magnet [3].

\subsection{YBCO coil design}

The YBCO coated conductor has a tape-like shape. There are two types of $\mathrm{YBCO}$ coil windings: solenoid and double pancake coils.

Solenoid coils are wound in spiral and offers the advantages of having high wire density and minimal connections between superconductors. However, the coated conductors on the edge of the coil are deformed and are subject to stress. Furthermore in case of damage, repair on part of a coil alone is not possible.

A double pancake coil is made of two single pancake coils which are wound vertically from the coil bobbin. The innermost layers of the coils are connected to each other by soldering. To make a large coil, several double pancake coils are stacked. Damage can easily be repaired by replacing damaged coils with a spare double pancake coil.

Considering the cost of YBCO conductors and the risk of coil failure, it was decided to make double pancake coil units and stack them. The magnetic field created by the coil is not uniform. Therefore, each YBCO coated conductor has a different magnetic field. Only a part of the double pancake coil unit reaches the critical current, limiting overall coil performance. Magnetic fields may be enhanced by replacing a coil unit which has reached the critical current with another coil unit made of high performance YBCO coated conductors.

Each double pancake coil should be packed in a metal
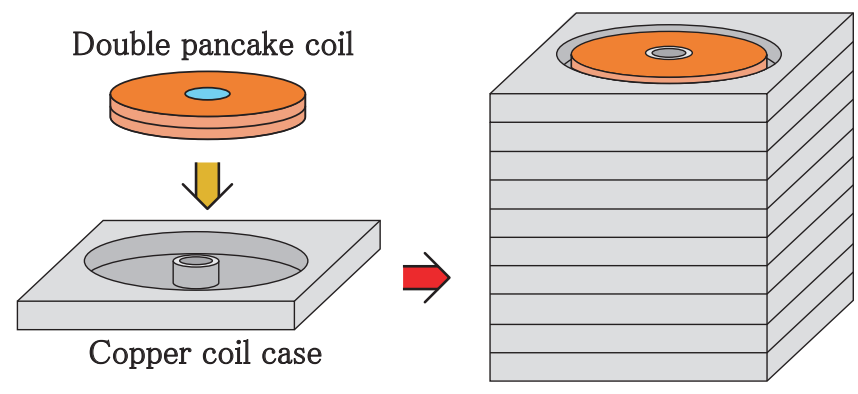

Stacked coil

Fig. 9 Fabrication process of the stacked YBCO coil

case. The metal cases increases thermal conductivity of the unit coils and facilitates stacking. Increment of current density is important to create high magnetic flux density. Therefore, the coil case must be thin, and is half-open (see Fig. 9).

The specifications of the each double pancake coil unit are listed in Table 2. Creating a magnetic flux density of 5 $\mathrm{T}$ means that some YBCO coated conductors in the stacked coil experience $5 \mathrm{~T}$ or much stronger magnetic flux density. The operational temperature, as mentioned above was approximately $50 \mathrm{~K}$. The critical current of a commercial YBCO coated conductor at $50 \mathrm{~K}$ in $5 \mathrm{~T}$ is estimated to be 80 A [4].

With a critical current of $80 \mathrm{~A}$, numerical simulation indicates that ten unit double pancake coils are necessary to create $5 \mathrm{~T}$. The simulation area is illustrated in Fig. 10

Table 2 Specifications of the double pancake coil units

\begin{tabular}{cc}
\hline Winding & Circular double-pancake winding \\
Inner / Outer diameter & $50 \mathrm{~mm} / 130 \mathrm{~mm}$ \\
Turns & 672 turns $(336$ turns $\times 2)$ \\
Wire length & $196 \mathrm{~m}(98 \mathrm{~m} \times 2)$ \\
Insulator & Polyimide tape \\
Inductance & $40 \mathrm{mH}$ \\
\hline Coil formation & Winding $($ impregnated $)+$ Copper coil case \\
Outer dimensions & $155 \mathrm{~mm} \times 155 \mathrm{~mm} \times 10 \mathrm{~mm}$
\end{tabular}

Detail of thickness direction $9 \mathrm{~mm}$ for winding setup, $1 \mathrm{~mm}$ for copper case

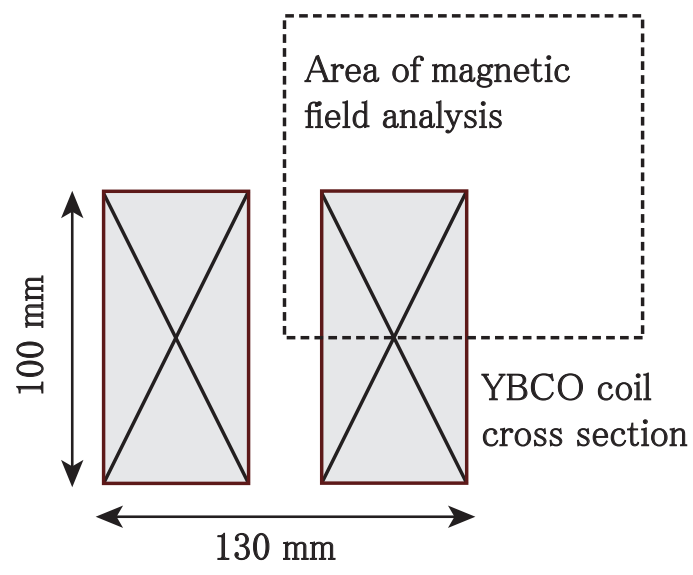

Fig. 10 Area of magnetic field analysis for the stacked coil 


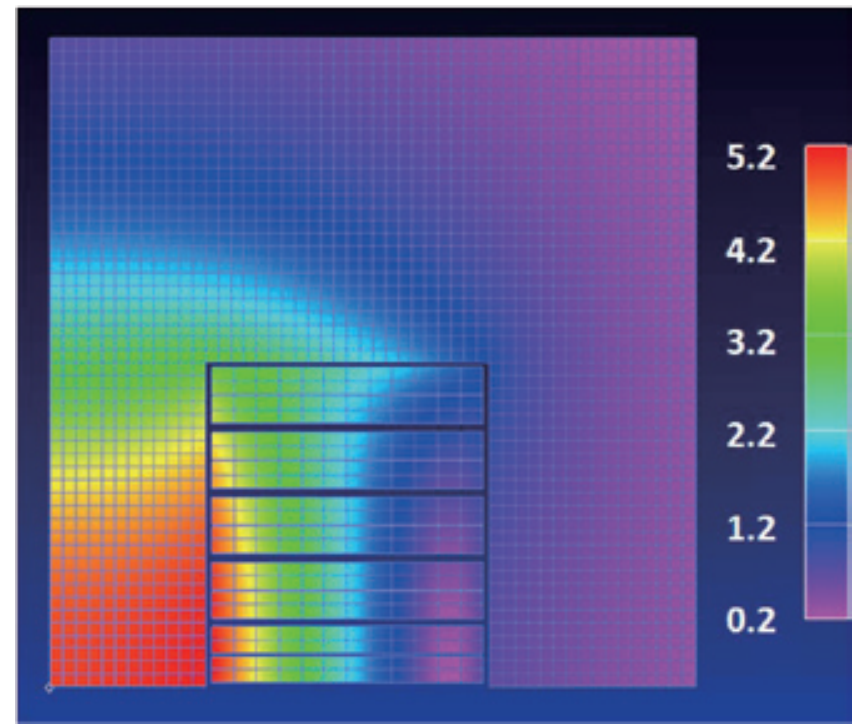

Fig. 11 Magnetic field distribution inside and around the stacked coil (one-fourth section model)

and the magnetic field distribution around and inside the coil is shown in Fig. 11.

The performance of coated conductors is not uniform. Whereas it is uncertain whether these stacked coils can create a magnetic flux density of $5 \mathrm{~T}$ at $50 \mathrm{~K}$ it is very probable that $5 \mathrm{~T}$ can be achieved at $40 \mathrm{~K}$.

Critical current measurements of the entire YBCO coated conductors, used in the coil, are essential to estimate the strict critical current of the coil. Since there are variations in coated conductor performance, this form of complete measurement is both time consuming and very costly. Consequently establishment of a simple and easy YBCO coil design method is also an important topic for research.

\subsection{Thermal design of the YBCO magnet}

An advantage of cryocooler cooling is that it is liquid helium free and easy to operate. Second stage cryocoolers are often used for cooling HTS applications. As the name implies, second stage cryocoolers have two cold stages. The first has large cooling power for high temperatures of around $100 \mathrm{~K}$ to $30 \mathrm{~K}$. The second stage has smaller cooling power for lower temperatures under $20 \mathrm{~K}$. Superconductors are cooled mainly by second stage cooling whereas the first stage is generally used for radiation shields. Radiation shields are the thin metal cases covering the superconductors which reduce heat leakage from the outer vessel.

An operational temperature of $50 \mathrm{~K}$ is fairly high even by HTS magnet standards. Such high temperatures mean that single stage cryocooler-cooling can be used with a radiation shield-less structure. A schematic drawing of the YBCO magnet is shown in Fig. 12. Without radiation shields, the distance between the coil and the inner surface of the vessel can be shortened. In the case of Maglev trains, that not only means downsizing of the magnet itself, but also increases interlinkage magnetic flux to the ground coil. Interlinkage magnetic flux generates levitation and propulsion force. Therefore, the value of magnetic flux den-

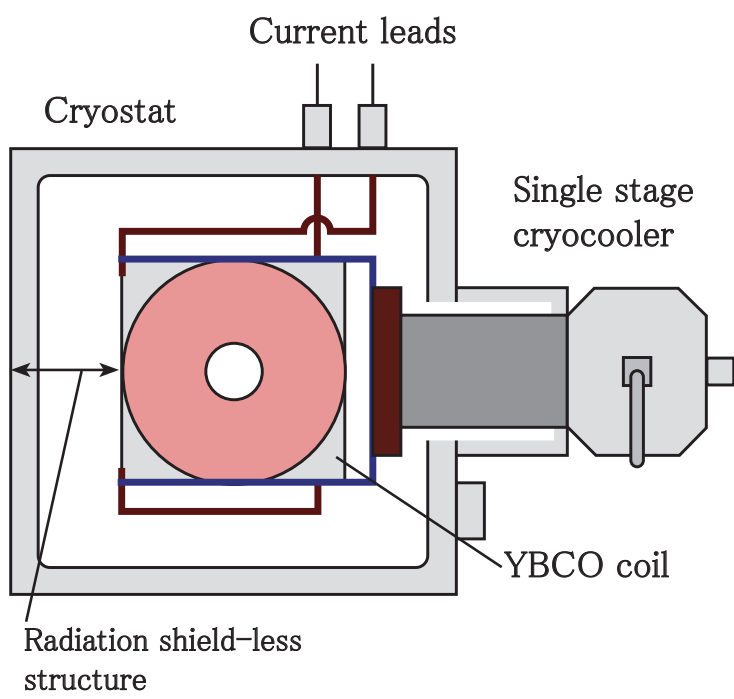

Fig. 12 Schematic drawing of the YBCO magnet

sity itself created by the superconducting magnet becomes unimportant. In other words, if the distance between the superconducting coil and the ground coil shortens, smaller superconducting coils are enough to create levitation and propulsion force. The total length of the superconductor decreases and the cost of the coil can be reduced.

Estimation of the total heat leakage of the YBCO magnet indicated that it was possible to use a single stage cryocooler. There are three types of heat leakage from the magnet: radiation, thermal conduction through the coil supports, and Joule heating from the current leads.

Theoretical radiation heat leakage and experimentally obtained radiation values do not correlate when the magnet has a complicated structure. Consequently radiation was estimated using the following equation based on experimentally measured radiation heat leakage.

$$
Q_{\text {radiation }}=A \alpha \frac{\left(T_{H}{ }^{4}-T_{L}^{4}\right)}{\left(300^{4}-77^{4}\right)}
$$

Where $T_{H}$ represents hotter temperatures, $T_{L}$ colder temperatures, $\mathrm{A}$ is the surface area and $\alpha$ is the experimental radiation heat leakage value from $300 \mathrm{~K}$ to $77 \mathrm{~K}$. Radiation heat leakage was estimated at $1.5 \mathrm{~W}$ using equation (1).

Coil supports are mainly made of low thermal conductivity and high strength material (e.g. grass fiber reinforced plastic or GFRP). Therefore, heat leakage through the coil supports was estimated to be approximately $2.0 \mathrm{~W}$ even though the magnet in this study was designed to be quite strong.

The heat leakage from current leads consists of Joule heating and thermal conduction. Thin current leads reduce thermal conduction, however Joule heating increases. Work on current lead optimization has already been carried out, and it is considered that heat leakage from $300 \mathrm{~K}$ to $50 \mathrm{~K}$ is approximately $0.1 \mathrm{~W}$ per ampere [5]. With extra current capacity, the current lead was designed for $100 \mathrm{~A}$, hence the heat leakage would be $10.0 \mathrm{~W}$.

There may also be some heat leakage from the measurement wires. Overall heat leakage was therefore estimated at $14.0 \mathrm{~W}$ as shown in Table 3 . Single stage cryocool- 
Table 3 Prospect of heat leak to the magnet

\begin{tabular}{l|c}
\hline Radiation & $1.5 \mathrm{~W}$ \\
\hline $\begin{array}{l}\text { Conduction through } \\
\text { the supports }\end{array}$ & $2.0 \mathrm{~W}$ \\
\hline $\begin{array}{l}\text { Conduction and Joule } \\
\text { heating from the cur- } \\
\text { rent leads }\end{array}$ & $10.0 \mathrm{~W}$ \\
\hline $\begin{array}{l}\text { Conduction through } \\
\text { measuring cables }\end{array}$ & $0.5 \mathrm{~W}$ \\
\hline \hline Total heat leakage & $14.0 \mathrm{~W}$ \\
\hline
\end{tabular}

ers with $14.0 \mathrm{~W}$ cooling power at $50 \mathrm{~K}$ are widely available.

\section{Conclusion}

Cyanoacrylate resin was investigated as a potential impregnation material. The bonding strength of cyanoacrylate is several times weaker than that of epoxy resin. Actual YBCO coil impregnation tests demonstrated cyanoacrylate does not damage YBCO coated conductors. Cyanoacrylate impregnation could be applicable to non-circular coils such as race-track coils, because cyanoacrylate resin still has bonding strength at cryogenic temperatures, unlike paraffin or wax.

The final target of this research is to apply the YBCO coated conductor technology to on-board Maglev train magnets. A small YBCO magnet is developed with the capacity to create a magnetic flux density of $5 \mathrm{~T}$. A numerical simulation and $\mathrm{YBCO}$ coated conductor specification test results indicate that $5 \mathrm{~T}$ is achievable with the coated conductor at high temperatures of approximately $50 \mathrm{~K}$. An operational temperature of $50 \mathrm{~K}$ means the structure does not require radiation shields and the magnet can be downsized.

This work was financially supported by the Japanese Ministry of Land, Infrastructure, Transport and Tourism.

\section{References}

[1] Y. Yanagisawa, H. Nakagome, T. Takematsu, T. Takao, N. Sato, M. Takahashi and H. Maeda "Remarkable weakness against cleavage stress for YBCO-coated conductors and its effect on the YBCO coil performance," Physica C, Vol.471, pp.480-485, 2011.

[2] T. Takematsu, R. Hu, T. Takao, Y .Yanagisawa, H .Nakagome, D. Uglietti, T. Kiyoshi, M. Takahashi and H. Maeda, "Degradation of the performance of a YBCOcoated conductor double pancake coil due to epoxy impregnation," Physica C, Vol.470, pp.674-677, 2010.

[3] K. Nagashima, M. Ogata, K, Mizuno, Y. Arai, H, Hasegawa and T. Sasakawa, "Study on Component of Superconducting magnet for Maglev Using High-temperature Superconducting Wire Based on Rare Earth Barium Copper Oxide," RTRI Report, Vol.25, No.3, pp.17-22, 2011 (in Japanese).

[4] M. Ogata, Y. Miyazaki, H. Hasegawa, T. Sasakawa and K. Nagashima, "Basic study of HTS magnet using $2 \mathrm{G}$ wires for maglev train," Physica C, Vol.470, pp.17821786, 2010.

[5] Martin N. Wilson, Superconducting Magnets. Oxford science, U.S.A., pp. 256-266, 1983. 